\title{
In patients eligible for meniscal surgery who first receive physical therapy, multivariable prognostic models cannot predict who will eventually undergo surgery
}

\author{
Julia C. A. Noorduyn ${ }^{1}$ (D) M. M. H. Teuwen ${ }^{1,2}$ (D) - V. A. van de Graaf ${ }^{1,8}$ (D) N. W. Willigenburg ${ }^{1}$ (D) M. Schavemaker ${ }^{3}$ (D)

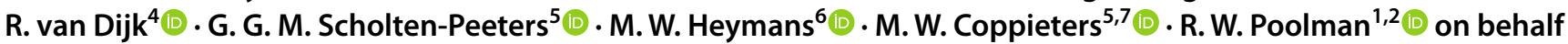 \\ of the ESCAPE Research Group
}

Received: 16 September 2020 / Accepted: 20 January 2021 / Published online: 7 February 2021

(c) The Author(s) 2021

\begin{abstract}
Purpose Although physical therapy is the recommended treatment in patients over 45 years old with a degenerative meniscal tear, $24 \%$ still opt for meniscal surgery.

The aim was to identify those patients with a degenerative meniscal tear who will undergo surgery following physical therapy. Methods The data for this study were generated in the physical therapy arm of the ESCAPE trial, a randomized clinical trial investigating the effectiveness of surgery versus physical therapy in patients of 45-70 years old, with a degenerative meniscal tear. At 6 and 24 months patients were divided into two groups: those who did not undergo surgery, and those who did undergo surgery. Two multivariable prognostic models were developed using candidate predictors that were selected from the list of the patients' baseline variables. A multivariable logistic regression analysis was performed with backward Wald selection and a cut-off of $p<0.157$. For both models the performance was assessed and corrected for the models' optimism through an internal validation using bootstrapping technique with 500 repetitions.

Results At 6 months, 32/153 patients (20.9\%) underwent meniscal surgery following physical therapy. Based on the multivariable regression analysis, patients were more likely to opt for meniscal surgery within 6 months when they had worse knee function, lower education level and a better general physical health status at baseline. At 24 months, $43 / 153$ patients (28.1\%) underwent meniscal surgery following physical therapy. Patients were more likely to opt for meniscal surgery within 24 months when they had worse knee function and a lower level of education at baseline at baseline. Both models had a low explained variance (16 and 11\%, respectively) and an insufficient predictive accuracy.

Conclusion Not all patients with degenerative meniscal tears experience beneficial results following physical therapy. The non-responders to physical therapy could not accurately be predicted by our prognostic models.
\end{abstract}

Level of evidence III.

Keywords Meniscus $\cdot$ Physical therapy $\cdot$ Prognostic model $\cdot$ Knee

Members of "The ESCAPE Research Group" are listed in

Acknowledgement section.

Julia C. A. Noorduyn

j.c.a.noorduyn@olvg.nl

1 Department of Orthopaedic Surgery, Joint Research, OLVG Amsterdam, Oosterpark 9, 1091 AC Amsterdam, The Netherlands

2 Department of Orthopaedics, Leiden University Medical Centre, Leiden, The Netherlands

3 Department of Radiology, Dijklander Ziekenhuis, Hoorn, The Netherlands

4 Department of Radiology, Isala, Zwolle, The Netherlands
5 Faculty of Behavioural and Movement Sciences, Department of Human Movement Sciences, Amsterdam Movement Sciences, Vrije Universiteit Amsterdam, Amsterdam, The Netherlands

6 Department of Epidemiology and Biostatistics, VU University Medical Centre, Amsterdam, The Netherlands

7 Menzies Health Institute Queensland, Griffith University, Brisbane \& Gold Coast, Australia

8 Department of Orthopeadic surgery, Antonius ziekenhuis, Nieuwegein, The Netherlands 


\section{Introduction}

Current guidelines state that physical therapy is the preferred first-line treatment in patients over 45 years of age with a degenerative meniscal tear $[15,19]$. These guidelines are based on several randomized clinical trials (RCT) which demonstrated no clinically superiority of meniscal surgery over physical therapy in this population [2, 4-7, $9,12,16,17,21,25]$. However, not all patients experience beneficial results from physical therapy. An average of $24 \%$ (between 1.9 and $36 \%$ ) of patients randomized to physical therapy still opt for meniscal surgery following conservative management [1].

Little information is available to predict at baseline the outcome of physical therapy in patients with a meniscal tear in both primary and secondary care, when a patient is referred by a general practitioner to an orthopedic surgeon. In secondary care, the patient and orthopedic surgeon may choose for surgical management, or to start a physical therapist-led exercise program. Patients rely on the orthopedics surgeons' expertise to help decide on their treatment pathway. However, recent research showed that orthopedic surgeons were not able to predict whether patients would benefit from either meniscal surgery or physical therapy [20].

Patients with shorter symptom duration and more knee pain at baseline are more likely to undergo meniscal surgery following physical therapy [8]. However, this study did not report the accuracy of the association model. Also, the potential predictors with a continuous outcome were dichotomized before the logistic regression analysis. This makes it difficult to reliably predict which patients will undergo surgery following physical therapy based on the current literature.

Therefore, the aims of this study were to develop and internally validate multivariable clinical prognostic models to identify those patients who will undergo surgery following physical therapy.

\section{Materials and methods}

Two prediction models were developed and internally validated for the outcome: meniscal surgery at 6 and 24 months after initial physical therapy in middle aged and older patients with a symptomatic degenerative meniscal tear. The data for this study were generated in the physical therapy arm of the ESCAPE trial. The ESCAPE trial was a multi-centre RCT comparing meniscal surgery with physical therapy in patients over 45 years old with a degenerative meniscal tear [21]. The Medical Research
Ethics Committees (MEC-U; NL44188.100.13) approved the ESCAPE trial. The trial was registered at clinincaltrials.gov (NCT01850719) and The Netherlands Trial Register (NTR3908). The current study was reported according to the transparent reporting of a multivariable prediction model for individual prognosis or diagnosis (TRIPOD) statement [11].

\section{Participants}

Patients aged between 45 and 70 years were referred by a general practitioner to the orthopaedic surgeon for diagnosis and treatment of their knee symptoms. All patients were diagnosed with a symptomatic degenerative meniscal tear. Besides that patients presented with symptoms, such as pain, the meniscal tear was confirmed on MRI. All patients were eligible for surgery and conservative treatment under the existing guidelines at the time. Patients who experienced a locked knee were excluded since this is an indication for surgery. In the ESCAPE-trial, patients were randomized to either immediate surgery or physical therapy. The physical therapy program consisted of a physical therapist-led standardized incremental exercise program containing of coordination/balance, closed kinetic chain strengths and cardiovascular exercises (see Appendix 1). The program was designed for 8 weeks with a total of 16 treatment sessions, each with a duration of $30 \mathrm{~min}$ [21]. As the Dutch health insurance does not cover PT, all 16 sessions were reimbursed by our research grant. If knee symptoms persisted following the physical therapy program (e.g., knee pain, limitations in daily activities or mechanical dysfunction), additional physical therapy sessions could be attended (not reimbursed by the study) or meniscal surgery, depending on a shared decision after consultation with the orthopaedic surgeon. All participants provided written informed consent [22].

\section{Outcome}

The outcome was opting for meniscal surgery following physical therapy. Patients who attended less than six physical therapy sessions were excluded for the analyses. At both the 6 months and the 24 months follow up, the binary outcome was whether patients who were randomized to physical therapy treatment had undergone delayed surgery (1) or not $(0)$.

\section{Candidate predictor selection}

From an extensive list of baseline variables assessed within the ESCAPE trial, candidate predictors were selected using a combination of three methods. First, a literature search was conducted to identify factors associated with the outcome after physical therapy treatment in patients with a meniscal 
tear. The search strategy can be found in Appendix 2. Second, an electronic survey was sent to an expert panel of orthopaedic surgeons $(N=24)$, physical therapists $(N=22)$ and patients $(N=10)$ who were involved in the ESCAPE trial to identify the most relevant prognostic factors for physical therapy according to their opinion. The survey consisted of an extensive list of baseline variables assessed within the ESCAPE trial. Third, a univariable logistic regression analysis was conducted to include additional potential predictors in the prognostic models.

The selection of potential predictors contained of patients' demographics, patient reported outcome (PROM) measures and radiographic information on MRI and radiograph. Demographic information included age, sex, level of education and body mass index (BMI). PROMs consisted of the International Knee Documentation Committee Subjective Knee Form for knee function, the visual analogue scale for pain during activities, the RAND-36 physical component scale for general physical health and patients' expectation on pain relieve with physical therapy at 6 months following physical therapy on a 7-point Likert scale, ranging from pain will be severely worse (1) to pain will be relieved completely (7). The radiographic information consisted of the Kellgren-Lawrence score for osteoarthritis, determined on a standing radiograph in posterior-anterior direction. The information on MRI consisted of the tear location (medial, lateral or both) and the tear type according to the International Society of Arthroscopy, Knee Surgery and Orthopaedic Sports (ISAKOS) (longitudinal vertical, horizontal, radial, vertical flap, complex/degenerative, not able to classify).

A final selection of 10 potential predictors was made by the principle researchers of this study (JCAN, VAG, NWW and RWP). Then the potential predictors were ranked to decide which will be included in the model, based on the 10 events per potential predictor rule [11]. (see Appendix 3). A more detailed description of the selection procedures can be found in Appendix 2.

\section{Statistical analysis}

A complete case analysis was performed since the percentage of missing values was lower than $10 \%$ [10, 18, 24]. Before building the model, underlying assumptions of linearity between independent continuous variables and the outcome and multicollinearity for the potential predictors were checked [3].

Two prognostic models were developed, one for the outcome at 6 months and one at 24 months, using a multivariable logistic regression analysis with Backward Wald Selection and a cut-off of $p<0.157$ [11, 18]. The performance of the models was assessed by the explained variance, the calibration and the discriminative ability of the models [3,
$11,18]$. The explained variance is determined by the Nagelkerke's $R^{2}$ statistic, with a larger $R^{2}$ indicating that a larger proportion of the variance can be explained by the model. Calibration, also called goodness of fit, was assessed by the Hosmer and Lemeshow test and calibration slope of the calibration plots $[3,18]$. A good model fit was established when the Hosmer and Lemeshow test was not significant. The calibration slope indicates an over-, smaller than 1 , or underfitting, larger than 1 , of the model. The discriminative ability of the models was determined by the Area under the Curve (AUC) $[11,18]$. An AUC between 0.6 and 0.8 was considered acceptable and a value of 0.8 or higher represents good discriminative ability of the model [11]. All statistical analyses were performed using IBM SPSS, version 22 (IBM Corp, Armonk, NY, USA).

To correct for the optimism in the prognostic model the final model was internally validated using bootstrapping technique with 500 repetitions $[3,11]$ The statistical software R-studio version 1.2.1335 (R-studio Inc., Boston, MA, USA) was used for the internal validation,. The correction factor from the bootstrapping was applied to the regression coefficients and performance measures.

\section{Results}

A total of 161 patients were allocated to physical therapy. Eight patients were excluded prior to data analysis because they attended less than six physical therapy sessions. At 6 months, 32 patients (20.9\%) had undergone meniscal surgery. At 24 months, an additional 11 patients had undergone meniscal surgery, resulting in a total of 43 patients (28.1\%). The baseline characteristics of both groups are presented in Table 1.

In the $28.1 \%$ of patients who underwent a meniscal surgery, 8 patients $(18.6 \%)$ expected no relieve in pain (score $1-4)$ following physical therapy. In the patients who did not undergo surgery, 10 patients $(9.1 \%)$ expected no relieve in pain following physical therapy.

\section{Multivariable regression analyses}

A complete case analysis was performed for both models with 153 cases. The model at 6 months confirmed all three candidate predictors as significant prognostic predictors: patient-reported knee function, education level and general physical health. Patients with worse knee function at baseline, a lower level of education and better self-reported general physical health had a higher probability of undergoing meniscal surgery. The results of the multivariable regression analyses, model performance measures and internal validation are presented in Table 2. The explained variance of the model was $16 \%$, indicating that the predicted outcome can 
Table 1 Baseline characteristics per group for the models at 6 and 24 months

\begin{tabular}{|c|c|c|c|c|c|c|}
\hline & \multicolumn{3}{|l|}{ Model at 6 months } & \multicolumn{3}{|l|}{ Model at 24 months } \\
\hline & No meniscal surgery & $\begin{array}{l}\text { Meniscal } \\
\text { surgery after } \\
\text { PT }\end{array}$ & $p$ value $^{a}$ & No meniscal surgery & $\begin{array}{l}\text { Meniscal } \\
\text { surgery after } \\
\text { PT }\end{array}$ & $p$ value $^{a}$ \\
\hline Demographics & $N=121$ & $N=32$ & & $N=110$ & $N=43$ & \\
\hline Age in years & $57.2(6.8)$ & $57.4(7.0)$ & n.s. & $57.7(7.0)$ & $56.4(6.7)$ & n.s. \\
\hline Women & $63(52.1 \%)$ & $16(50.0 \%)$ & n.s. & $58(52.7 \%)$ & $21(48.8 \%)$ & n.s. \\
\hline Body Mass Index & $27.0(4.0)$ & $27.6(3.9)$ & n.s. & $27.1(4.1)$ & $27.2(3.7)$ & n.s. \\
\hline Education level (score is $1-7)^{\mathrm{b}}$ & $4.8(1.8)$ & $3.8(1.7)$ & 0.05 & $4.8(1.8)$ & $4.0(1.8)$ & 0.02 \\
\hline Smoking (yes) & $16(13.2 \%)$ & $3(9.4 \%)$ & n.s. & $14(12.7 \%)$ & $5(11.6 \%)$ & n.s. \\
\hline \multicolumn{7}{|l|}{ Patient reported outcomes } \\
\hline Pain during activities (NRS; 0-100) & $56.1(22.4)$ & $67.9(21.1)$ & 0.02 & $56.1(22.2)$ & $64.9(22.5)$ & n.s. \\
\hline Knee function (IKDC; 0-100) & $48.8(14.1)$ & $39.6(13.6)$ & $<0.01$ & $48.9(14.2)$ & $41.7(14.2)$ & 0.05 \\
\hline Physical health (Rand-36 PCS; 0-100) & $38.7(8.7)$ & $36.2(8.1)$ & n.s. & $38.8(8.7)$ & $36.5(8.4)$ & n.s. \\
\hline \multicolumn{7}{|l|}{ Patient expectation } \\
\hline No pain relieve within 6 months & $11(9.1 \%)$ & $7(21.9 \%)$ & n.s. & $10(9.1 \%)$ & $8(18.6 \%)$ & n.s. \\
\hline Pain relieve within 6 months & $110(90.9 \%)$ & $25(78.1 \%)$ & & $100(90.9)$ & $35(81.4 \%)$ & \\
\hline \multicolumn{7}{|l|}{ Imaging results } \\
\hline OA score on radiograph (KL classification) ${ }^{\mathrm{d}}$ & & & n.s. & & & n.s. \\
\hline 0: No OA & $12(9.9 \%)$ & $2(6.3 \%)$ & & $5(4.5 \%)$ & $0(0 \%)$ & \\
\hline 1: Doubtful & $55(45.5 \%)$ & $16(50 \%)$ & & $37(33 \%)$ & $10(21.7 \%)$ & \\
\hline 2: Minimal OA & $45(37.2 \%)$ & $8(25 \%)$ & & $35(31.3 \%)$ & $9(19.6 \%)$ & \\
\hline 3: Moderate OA & $2(1.7 \%)$ & $2(6.3 \%)$ & & $6(5.4 \%)$ & $1(2.2 \%)$ & \\
\hline 4: Severe $\mathrm{OA}^{\mathrm{e}}$ & $0(0 \%)$ & $0(0 \%)$ & & $0(0 \%)$ & $0(0 \%)$ & \\
\hline Affected meniscus ${ }^{\mathrm{f}}$ & & & n.s. & & & n.s. \\
\hline Medial & $93(76.9 \%)$ & $25(78.1 \%)$ & & $86(78.2 \%)$ & $32(74.4 \%)$ & \\
\hline Lateral & $19(15.7 \%)$ & $5(15.6 \%)$ & & $17(15.5 \%)$ & $7(16.3 \%)$ & \\
\hline Both & $9(7.4 \%)$ & $2(6.3 \%)$ & & $7(6.4 \%)$ & $4(9.3 \%)$ & \\
\hline
\end{tabular}

Data are presented as $n(\%)$ or mean (SD)

NRS numeric rating scale, higher score indicates more pain, IKDC International Knee Documentation Committee Subjective Knee Form, higher score indicates better knee function, RAND-36 PCS physical component score of the RAND-36 questionnaire, higher score indicates better physical health status, $O A$ osteoarthritis, $K L$ Kellgren-Lawrence classification of knee osteoarthritis, n.s. not significant

${ }^{\text {a }}$ Statistical differences between the surgery after PT group and no meniscal surgery group was assesses by an independent-sample $T$ test for continues data, or a $\chi^{2}$ test for binary and categorical data. $p$ values $\leq 0.05$ were considered significant

${ }^{\mathrm{b}}$ Education level measured according to the International Standard Classification of Education (ISCED) score ranges from 1 to 7 with a higher score indicating higher level of education

${ }^{c}$ Expectation of the pain score, 1 = pain will get severely worse and $6=$ pain will be relieved completely

${ }^{\mathrm{d}}$ Grade of knee osteoarthritis was assessed by X-ray using the Kellgren and Lawrence scale (K\&L)

ePatients with a KL classification of 4 on the baseline X-ray were excluded from the trial

${ }^{\mathrm{f}}$ Location of tear was assessed by magnetic resonance imaging

be explained for $16 \%$ by the predictors. The Hosmer and Lemeshow test was 0.12 and the mean calibration 0.003 , indicating a good model fit. However, the calibration plot displayed an overestimation of the predicted outcomes for the model with a calibration slope of $<1$. The discriminative ability of the model was adequate with an AUC of 0.73 . Internal validation resulted in a correction factor for the initial model's optimism of 0.90 . The correction factor was applied to the regression coefficients and performance measures.
The model at 24 months confirmed that worse patientreported knee function and lower level of education were prognostic factors for undergoing meniscal surgery. Patients with worse knee function at baseline, a lower level of education had a higher probability of undergoing meniscal surgery. The results of the multivariable regression analyses, model performance measures and internal validation are presented in Table 2. The explained variance of the model was $11 \%$, indicating that the predicted outcome can be explained for $11 \%$ by the predictors. The Hosmer and Lemeshow test 
Table 2 Prognostic models for meniscal surgery after initial PT treatment at 6 and 24 months

\begin{tabular}{|c|c|c|c|c|}
\hline Predictor & Beta $^{a}$ & Adusted beta ${ }^{\mathrm{b}}$ & OR $(95 \% \mathrm{CI})$ & $p$ value $^{\mathrm{c}}$ \\
\hline \multicolumn{5}{|l|}{ Model at 6 months } \\
\hline Knee function ${ }^{\mathrm{d}}$ & -0.06 & -0.05 & $0.94(0.90-0.98)$ & 0.01 \\
\hline Education level $^{\mathrm{e}}$ & -0.25 & -0.23 & $0.78(0.62-0.99)$ & 0.04 \\
\hline General physical health ${ }^{\mathrm{f}}$ & 0.05 & 0.05 & $1.05(0.98-1.13)$ & 0.15 \\
\hline \multicolumn{5}{|l|}{ Model at 24 months } \\
\hline Knee function ${ }^{\mathrm{d}}$ & -0.03 & -0.03 & $0.97(0.94-1.00)$ & 0.03 \\
\hline Education level $^{\mathrm{e}}$ & -0.17 & -0.14 & $0.84(0.69-1.03)$ & 0.10 \\
\hline Model performance & $R^{2}$ & AUC & Mean calibration & $\mathrm{H} \& \mathrm{~L}$ \\
\hline \multicolumn{5}{|l|}{ Model at 6 months } \\
\hline Initial model & 0.16 & 0.73 & 0.003 & 0.12 \\
\hline After internal validation ${ }^{\mathrm{b}}$ & 0.14 & 0.71 & & \\
\hline \multicolumn{5}{|l|}{ Model at 24 months } \\
\hline Initial model & 0.11 & 0.68 & 0.001 & n.s \\
\hline After internal validation ${ }^{\mathrm{b}}$ & 0.09 & 0.66 & & \\
\hline
\end{tabular}

95\% CI 95\% confidence interval, OR odds ratio, $R^{2}$ Nagelkerke's $R^{2}, A U C$ area under the curve, $H \& L$ Hosmer and Lemeshow test, $n . s$. not significant

${ }^{a}$ Positive beta is indicative that a higher score results in a higher probability of undergoing a meniscal surgery; a negative coefficient indicates that this risk increased with lower score. Some multicollinearity between the predictors can explain apparent discrepancies with baseline (Table 1)

${ }^{\mathrm{b}}$ Regression coefficients and performance measures for the model at 6 months were multiplied by the shrinkage factor of 0.90 retrieved from internal validation

Regression coefficients and performance measures for the model at 24 months were multiplied by the shrinkage factor of 0.82 retrieved from internal validation

${ }^{\mathrm{c}} p$ values lower than 0.157 are considered significant

${ }^{\mathrm{d}}$ Knee function measured with the International Knee Documentation Committee Subjective Knee Form (IKDC) score ranges from 0 to 100, a higher score indicates better knee function

${ }^{\mathrm{e}}$ Education level measured according to the International Standard Classification of Education (ISCED) score ranges from 1 to 7 with a higher score indicating higher level of education

${ }^{\mathrm{f}}$ General physical health measures with the RAND-36 Physical Component Score. Scores ranges rom 0-100, higher score indicates better health status

was 0.48 and the mean calibration 0.002, indicating a good model fit. However, the calibration plot displayed an overestimation of the predicted outcomes for the model with a calibration slope of $<1$. The discriminative ability of the model was adequate with an AUC of 0.68. Internal validation resulted in a correction factor for the initial model's optimism of 0.82 for the regression coefficients and performance measures.

\section{Discussion}

Two prognostic models were developed and internally validated to predict which patients will undergo meniscal surgery following physical therapy in patients with a degenerative meniscal tear. Patients who experienced a better general physical health status (for the 6 months model), and had worse knee function and lower education level (for both the 6 and 24 months model) were more likely to undergo meniscal surgery. However, both models showed a low explained variance and had an insufficient predictive accuracy. Therefore, external validation of these models is not useful since the models cannot be used in clinical practice.

Predicting treatment outcome for patients with a meniscal tear remains challenging. Recently, a study investigated the ability of orthopedic surgeons to predict the outcome of physical therapy and the outcome of surgery in patients over 45 years with a symptomatic meniscal tear [20]. Orthopedic surgeons received baseline characteristics of the patient including demographic information about employment, age and BMI, PROMs on pain, knee function and mechanical dysfunction, and MRI results on tear type and location, and radiograph information on level of knee osteoarthritis. Similar to the results of this study, they found that orthopedic surgeons were also unable to accurately predict which patient would benefit from physical therapy based on the baseline characteristics [20]. 
Multivariable prognostic prediction models have also been shown inaccurate in predicting the treatment outcome of initial meniscal surgery in a similar population [13]. The authors argued that treatment outcome cannot be accurately predicted in this population due to the combination of knee osteoarthritis and a meniscal tear, which is a common finding in middle aged and older patients [13]. Likewise, mild to moderate knee osteoarthritis was also found in our study in the majority of the patients [21]. This may have negatively impacted on the predictive ability of our models since patients might experience persistence of knee complaints due to overall degenerative knee pain instead of solely meniscal pain [13]. The current literature appears to report similar results as the current study which suggests that no subgroups can be identified who can benefit from surgery. The current study supports other literature that failed to identify subgroups of patients who can benefit from surgery [13]. The efficacy of physical therapy was not investigated in this study. However, given the absence of a clinically relevant benefit of surgery over conservative treatment $[2$, $4-7,9,12,16,17,21,25]$, and the lack of clear prognostic characteristics for treatment outcomes [13], clinicians should rely more on the current guidelines recommending physical therapy as the first-line treatment in patients with degenerative meniscal tears. [15, 19]

This study has some limitations. First, this study was not primary designed as a prognostic prediction model study. Using data collected within a RCT is suitable to develop prognostic models [11]. Nevertheless, it was a disadvantage that the variables, available for the development of the models, did not include some of the variables that were previously shown to be associated with the outcome. For instance, from the current literature the variable duration of symptoms was selected as prognostic factor [8]. However, duration of symptoms was not assessed in our study population and could therefore not be included in the model [23]. Second, the amount of candidate predictors was determined using the rule of 10 events per potential predictor. Although this is an accepted method and recommended in the TRIPOD statement, some researchers suggest that the rule of thumb is too simplistic to determine an adequate sample size for multivariable prognostic models with a binary outcome [14]. In our study the sample size and amount of events was fixed since data were used that were collected within the ESCAPE trial. Therefore the amount of candidate predictors was determined on our sample size, instead of vice a versa. Nevertheless, the results of this study meet the criteria, a shrinkage factor of $\geq 0.9$ that represents a small optimism in predictor effect estimates and a small absolute difference of $\leq 0.05$ in the model's initial and adjusted Nagelkerke's $R^{2}$, that Riley et al. [14] proposed for an adequate sample size. Last, the prescribed PT treatment was a standardized incremental exercise protocol.
With the current available evidence, it is impossible to identify which patient will require surgery following physical therapy. Instead, clinicians should recommend physical therapy as the first-line treatment for patients with degenerative meniscal tears, following the current guidelines $[15,19]$

\section{Conclusion}

With this study, the course of conservative treatment could not be predicted and patients who are likely to undergo meniscal surgery in the short (i.e., 6 months) and long term (i.e., 24 months) following physical therapy could not be identified. Therefore, these models should not be externally validated and not used in clinical practice. Future research should focus on identifying specific prognostic factors for treatment selection, surgery or physical therapy, in this population.

Supplementary Information The online version contains supplementary material available at https://doi.org/10.1007/s00167-021-06468-0.

Acknowledgements We thank all participants of the ESCAPE trial for their contribution in the study and the participating centers for their help in this study.

Escape Research Group Collaborators:

Scholtes VAB1, Mutsaerts ELAR1, Wolkenfelt J1, Krijnen MR1, van Deurzen DFP1, Moojen DJF1, Bloembergen CH1, de Gast A2, Snijders T2, Halma JJ2, Saris DBF3, Wolterbeek N4, Neeter C5, Kerkhoffs GMMJ6, Peters RW7, van den Brand ICJB8, de Vos-Jakobs S8, Spoor AB8, Gosens T8, Rezaie W8,9, Hofstee DJ10, Burger BJ10, Haverkamp D11, Vervest AMJS12, van Rheenen TA12, Wijsbek AE12, van Arkel ERA13, Thomassen BJW13, Sprague S14, Mol BWJ15, van Tulder MW16, J. van der Kraan17

1: Department of Orthopaedic Surgery, Joint Research, OLVG, Amsterdam, The Netherlands. 2: Department of Orthopaedic Surgery, Clinical Orthopaedic Research Center, Diakonessenhuis, Utrecht, The Netherlands. 3: Department of Orthopaedic Surgery, UMC Utrecht, Utrecht, The Netherlands. 4: Department of Orthopaedic Surgery, St. Antonius Ziekenhuis, Utrecht, The Netherlands. 5: Fysiken Physical Therapy, Amsterdam, The Netherlands. 6: Department of Orthopaedic Surgery, Academic Medical Center, Amsterdam, The Netherlands. 7: Department of Trauma Surgery, Academic Medical Center, Amsterdam, The Netherlands. 8: Department of Orthopaedic Surgery, Elisabeth Tweesteden Ziekenhuis, Tilburg, The Netherlands. 9: Department of Orthopaedic Surgery, Onze Lieve Vrouw Ziekenhuis, Aalst, Belgium. 10: Department of Orthopaedic Surgery, Noordwest Ziekenhuisgroep, Alkmaar, The Netherlands. 11: Department of Orthopaedic Surgery, Xpert Orthopedie, Amsterdam, The Netherlands. 12: Department of Orthopaedic Surgery, Tergooi Hospital, Hilversum, The Netherlands. 13: Department of human and Technology, The Hague University of Applied Sciences, Den Haag, The Netherlands. 14: Division of Orthopaedic Surgery, Department of Surgery, Department of Health Research Methods, Evidence, and Impact, McMaster University, Hamilton, ON, Canada. 15: Department of Obstetrics and Gynaecology, School of Medicine, Monash University, Melbourne, Australia. 16: Department of Health Sciences and the EMGO+ Institute for Health and Care Research, Vrije Universiteit, Amsterdam, The Netherlands. 17: Dutch Patients Federation, Utrecht, The Netherlands. 
Author contributions All authors contributed to the study conception and design. Data collection and analysis were performed by: JN, MT. The first draft of the manuscript was written by JN and all authors commented on previous versions of the manuscript. All authors read and approved the final manuscript.

Funding VG, NW, JN, and RP received financial support from The Netherlands Organisation for Health Research and Development (in Dutch: ZonMw) for the submitted work; the Achmea Healthcare Foundation (in Dutch Stichting Achmea Gezonheidszorg fonds), Dutch Arthritis Society and the foundation of medical research at the OLVG, Amsterdam, the Netherlands; no financial relationships with any organisations that might have an interest in the submitted work in the previous three years; no other relationships or activities that could appear to have influenced the submitted work

\section{Compliance with ethical standards}

Conflict of interest All authors have completed the Unified Competing Interest form (available on request from the corresponding author) and declared.

Ethics approval The Medical Research Ethics Committees (MEC-U; (NL4418810013)) approved the ESCAPE trial. The trial was registered at clinincaltrials.gov (NCT03462134) and The Netherlands Trial Register (NL3709).

Informed consent All participants provided written informed consent.

Open Access This article is licensed under a Creative Commons Attribution 4.0 International License, which permits use, sharing, adaptation, distribution and reproduction in any medium or format, as long as you give appropriate credit to the original author(s) and the source, provide a link to the Creative Commons licence, and indicate if changes were made. The images or other third party material in this article are included in the article's Creative Commons licence, unless indicated otherwise in a credit line to the material. If material is not included in the article's Creative Commons licence and your intended use is not permitted by statutory regulation or exceeds the permitted use, you will need to obtain permission directly from the copyright holder. To view a copy of this licence, visit http://creativecommons.org/licenses/by/4.0/.

\section{References}

1. Abram SGF, Hopewell S, Monk AP, Bayliss LE, Beard DJ, Price AJ (2019) Arthroscopic partial meniscectomy for meniscal tears of the knee: a systematic review and meta-analysis. Br J Sports Med. https://doi.org/10.1136/bjsports-2018-100223

2. Berg B, Roos EM, Englund M, Kise NJ, Tiulpin A, Saarakkala S et al (2020) Development of osteoarthritis in patients with degenerative meniscal tears treated with exercise therapy or surgery: a randomized controlled trial. Osteoarthr Cartil 28:897-906

3. Field A (2013) Discovering statistics using IBM SPSS Statistics, 4th edn. SAGE Publications, London

4. Gauffin H, Tagesson S, Meunier A, Magnusson H, Kvist J (2014) Knee arthroscopic surgery is beneficial to middle-aged patients with meniscal symptoms: a prospective, randomised, singleblinded study. Osteoarthr Cartil 22:1808-1816

5. Herrlin SV, Wange PO, Lapidus G, Hallander M, Werner S, Weidenhielm L (2013) Is arthroscopic surgery beneficial in treating non-traumatic, degenerative medial meniscal tears? A five year follow-up. Knee Surg Sports Traumatol Arthrosc 21:358-364

6. Katz JN, Brophy RH, Chaisson CE, de Chaves L, Cole BJ, Dahm DL et al (2013) Surgery versus physical therapy for a meniscal tear and osteoarthritis. N Engl J Med 368:1675-1684

7. Katz JN, Shrestha S, Losina E, Jones MH, Marx RG, Mandl LA et al (2020) Five-year outcome of operative and nonoperative management of meniscal tear in persons older than forty-five years. Arthritis Rheumatol 72:273-281

8. Katz JN, Wright J, Spindler KP, Mandl LA, Safran-Norton CE, Reinke EK et al (2016) Predictors and outcomes of crossover to surgery from physical therapy for meniscal tear and osteoarthritis: a randomized trial comparing physical therapy and surgery. $\mathrm{J}$ Bone Joint Surg Am 98:1890-1896

9. Kise NJ, Risberg MA, Stensrud S, Ranstam J, Engebretsen L, Roos EM (2016) Exercise therapy versus arthroscopic partial meniscectomy for degenerative meniscal tear in middle aged patients: randomised controlled trial with two year follow-up. BMJ 354:i3740

10. Marshall AAD, Royston P, Holder RL (2010) Comparison of techniques for handling missing covariate data within prognostic modelling studies: a simulation study. BMC Med 10:16

11. Moons KG, Altman DG, Reitsma JB, Ioannidis JP, Macaskill P, Steyerberg EW et al (2015) Transparent reporting of a multivariable prediction model for individual prognosis or diagnosis (TRIPOD): explanation and elaboration. Ann Intern Med 162:W1-73

12. Noorduyn JCA, Glastra van Loon T, van de Graaf VA, Willigenburg NW, Butter IK, Scholten-Peeters GGM et al (2020) Functional outcomes of arthroscopic partial meniscectomy versus physical therapy for degenerative meniscal tears using a patientspecific score: a randomized controlled trial. Orthop J Sports Med. https://doi.org/10.1177/2325967120954392

13. Pihl K, Ensor J, Peat G, Englund M, Lohmander S, Jorgensen U et al (2019) Wild-goose chase, no predictable patient subgroups who benefit from meniscal surgery: patient-reported outcomes of 641 patients 1 year after surgery. Br J Sports Med. https://doi. org/10.1136/bjsports-2018-100321

14. Riley RD, Snell KI, Ensor J, Burke DL, Harrell FEJ, Moons KGM et al (2018) Minimum sample size for developing a multivariable prediction model: PART II - binary and time-to-event outcomes. Stat Med 38:20

15. Siemieniuk RAC, Harris IA, Agoritsas T, Poolman RW, Brignardello-Petersen R, Van de Velde S et al (2018) Arthroscopic surgery for degenerative knee arthritis and meniscal tears: a clinical practice guideline. Br J Sports Med 52:313

16. Sihvonen R, Paavola M, Malmivaara A, Itala A, Joukainen A, Kalske J et al (2020) Arthroscopic partial meniscectomy for a degenerative meniscus tear: a 5 year follow-up of the placebosurgery controlled FIDELITY (Finnish Degenerative Meniscus Lesion Study) trial. Br J Sports Med 54:1332-1339

17. Sonesson S, Kvist J, Yakob J, Hedevik H, Gauffin H (2020) Knee Arthroscopic surgery in middle-aged patients with meniscal symptoms: a 5-year follow-up of a prospective, randomized study. Orthop J Sports Med. https://doi.org/10.1177/2325967119893920

18. Steyerberg EW (2009) Dealing with missing values. In: Clinicial prediction models: a practical approach to development, validation, and updating, 1st edn. Springer, New York

19. van Arkel ERA, van Essen A, Koëter S, et al (2019) Artroscopie van de knie. Dutch Orthopedic Association guideline 2019. website Dutch Orthopedic Association: www. orthopeden.org.

20. van de Graaf VA, Bloembergen CM, Willigenburg NWP, Noorduyn JCAM, Saris D, Harris I et al (2019) Can even experienced orthopaedic surgeons predict who will benefit from surgery when patients present with degenerative meniscal tears? A survey of 194 orthopaedic surgeons who made 3880 predictions. Br J Sports Med. https://doi.org/10.1136/bjsports-2019-100567 
21. van de Graaf VA, Noorduyn JCA, Willigenburg NW, Butter IK, de Gast A, Mol BW et al (2018) Effect of early surgery vs physical therapy on knee function among patients with nonobstructive meniscal tears: the escape randomized clinical trial. JAMA 320:1328-1337

22. van de Graaf VA, Scholtes VA, Wolterbeek N, Noorduyn JC, Neeter C, van Tulder MW et al (2016) Cost-effectiveness of Early surgery versus conservative treatment with optional delayed meniscectomy for patients over 45 years with non-obstructive meniscal tears (ESCAPE study): protocol of a randomised controlled trial. BMJ Open 6:e14381

23. van de Graaf VA, Wolterbeek N, Mutsaerts EL, Scholtes VA, Saris DB, de Gast A et al (2016) Arthroscopic partial meniscectomy or conservative treatment for nonobstructive meniscal tears: a systematic review and meta-analysis of randomized controlled trials. Arthroscopy 32(1855-1865):e1854
24. Vergouwe Y, Royston P, Moons KGM, Altman DG (2010) Development and validation of a prediction model with missing predictor data: a practical approach. J Clin Epidemiol 63:9

25. Yim JH, Seon JK, Song EK, Choi JI, Kim MC, Lee KB et al (2013) A comparative study of meniscectomy and nonoperative treatment for degenerative horizontal tears of the medial meniscus. Am J Sports Med 41:1565-1570

Publisher's Note Springer Nature remains neutral with regard to jurisdictional claims in published maps and institutional affiliations. 